Review

\title{
Sequestration and Transport of Lignin Monomeric Precursors
}

\author{
Chang-Jun Liu *, Yu-Chen Miao and Ke-Wei Zhang \\ Biology Department, Brookhaven National Laboratory, Upton, NY 11973, USA \\ * Author to whom correspondence should be addressed; E-Mail: cliu@bnl.gov; \\ Tel.: +1-631-344-2966; Fax: +1-631-344-3407.
}

Received: 23 November 2010; in revised form: 13 January 2011 / Accepted: 17 January 2011 / Published: 18 January 2011

\begin{abstract}
Lignin is the second most abundant terrestrial biopolymer after cellulose. It is essential for the viability of vascular plants. Lignin precursors, the monolignols, are synthesized within the cytosol of the cell. Thereafter, these monomeric precursors are exported into the cell wall, where they are polymerized and integrated into the wall matrix. Accordingly, transport of monolignols across cell membranes is a critical step affecting deposition of lignin in the secondarily thickened cell wall. While the biosynthesis of monolignols is relatively well understood, our knowledge of sequestration and transport of these monomers is sketchy. In this article, we review different hypotheses on monolignol transport and summarize the recent progresses toward the understanding of the molecular mechanisms underlying monolignol sequestration and transport across membranes. Deciphering molecular mechanisms for lignin precursor transport will support a better biotechnological solution to manipulate plant lignification for more efficient agricultural and industrial applications of cell wall biomass.
\end{abstract}

Keywords: lignin; monolignols; monolignol glucosides; $\mathrm{ABC}$ transporters; vacuolar sequestration

\section{Introduction}

Lignin is the second most abundant terrestrial biopolymer after cellulose, accounting for approximately $30 \%$ of organic carbon in the biosphere [1]. It is a crucial component in preserving the structural integrity of plant cell wall, therefore, affording stiffness and strength of the stem of vascular plants; enabling transport of water and solutes through tubes in the vascular system; and physically 
protecting plants against pathogen infection and other environmental stresses [1,2]. However, its presence in cell wall greatly contributes to the wall's recalcitrance to hydrolysis, thus detrimental to the applications of cellulosic fibers for pulping and biofuel production [3-5]. Plant lignification is considered to occur in three stages: biosynthesis of monolignols in the cell, transport of monolignols to the cell wall, and dehydrogenative polymerization of monolignols within the cell wall. Although extensive studies have centered on lignin biosynthesis for many decades, several fundamental aspects on plant lignification remain elusive. One of the most intriguing yet least understood aspects concerns the intracellular sequestration of lignin precursors and their transport across the plasma membrane. In this review, we outline the hypothesized mechanisms for monolignol sequestration and transport, and describe the recent progresses in genetic, transcriptomic, proteomic, modern autoradiographic, and biochemical studies toward understanding the underlying molecular mechanisms for monolignol sequestration and transport across membranes. An improved knowledge of transport of monolignols will facilitate the efficient manipulation of plant ligninfication for utilization of lignocellulosic biomass.

\section{Results and Discussion}

\subsection{Monolignol biosynthesis and subcellular localization of the related enzymes}

Lignins are complex racemic aromatic heteropolymers derived mainly from three hydroxycinnamyl alcohols, i.e., p-coumaryl, coniferyl and sinapyl alcohols [2]. Biosynthesis of monolignols diverges from the shikimate pathway. Starting from phenylalanine, monolignol biosynthesis is sequentially catalyzed by about 10 enzymes [4], namely phenylalanine ammonia lyase (PAL), cinnamic acid 4hydroxylase $(\mathrm{C} 4 \mathrm{H})$, 4-hydroxycinnamoyl CoA ligase $(4 \mathrm{CL})$, hydroxycinnamoyl CoA:shikimate hydroxycinnamoyl transferase $(\mathrm{HCT})$, p-coumaroylshikimate 3'-hydroxylase $\left(\mathrm{C} 3^{\prime} \mathrm{H}\right)$, caffeoyl CoA $O$ methyltransferase (CCoAOMT), hydroxycinnamoyl CoA reductase (CCR), ferulic acid 5-hydroxylase (F5H), caffeic acid/5-hydroxyferulic acid O-methyltransferase (COMT), and (hydroxy)cinnamyl alcohol dehydrogenase (CAD) [1]. The activities of these enzymes lead to deamination, hydroxylation, transacylation, methylation, and reduction of phenylalanine, and transform it to the lignin monomeric precursors, p-coumaryl, coniferyl, and sinapyl alcohols (i.e., monolignols) [1,4]. Among these enzymes, three cytochrome $\mathrm{P} 450$ proteins, i.e., cinnamic acid 4-hydroxylase (C4H), pcoumaroylshikimate $3^{\prime}$-hydroxylase $\left(\mathrm{C}^{\prime} \mathrm{H}\right)$, and ferulic acid 5-hydroxylase $(\mathrm{F} 5 \mathrm{H})$ are membranebound proteins and associate with the outer surface of the endoplasmic reticulum by virtue of their $\mathrm{N}$ terminal membrane anchor [4]. Nevertheless, most other enzymes, such as PAL, 4CL, COMT, CCoAOMT and CAD in diverse species are soluble and localize within cytosol that were revealed by biochemical and immuno-cytochemical studies, although a few studies suggested that PAL and CAD may have different types of isoforms, and some of them may associate to the ER-Golgi derived vesicles [6,7]. For example, one tobacco PAL isoform, when fused with GFP protein, was found localized to microsomal fractions, where it co-localized with $\mathrm{C} 4 \mathrm{H}$, forming a potential metabolic channeling at the entry point of phenylpropanoid-lignin biosynthetic pathway [8]. 
The cytosolic localization of those biosynthetic enzymes suggests that monolignols are synthesized in the cytoplasm. After that, they need to be transported to the cell wall where they are oxidatively polymerized, and integrated into the secondary cell-wall matrix.

\subsection{Monolignol glucosylation and its potential roles in sequestration and transport of monolignols}

In gymnosperms and some angiosperm species, monolignols are often glycosylated to form 4-O- $\beta$ D-glucosides, namely, coniferin and syringin [9]. Assessing monolignol glucoside content in several dicot trees and gymnosperm species from different genera, Terazawa et al. found that the monolignol glucosides (coniferin) were present primarily in gymnosperm species [10]. Leinhos and Savidge also showed that coniferin present at high level within protoplasts made from the developing xylems of Jack pine (Pinus banksiana) and Eastern white pine (P. strobes) [9]. The coniferin content of the protoplasts can account for most, if not all, of the coniferin present in the tissue. This leads to the assumption that the high level-accumulated monolignol glucosides most likely are stored in vacuoles of cambial cells [11-14].

The possible presence of monolignol glucosides in vacuoles of differentiating conifer xylem cells led to the suggestion that at least in gymnosperms and some evolutionarily less-derived angiosperm species such as Magnoliaceae and Oleaceae families, monolignol glucosides may be the storage or transport forms of the monolignols; and the lignin biosynthesis and mobilization of the synthetic precursors in these species may be regulated by monolignol glucosides. The glucoconjugates of monolignols are possibly sequestrated first from cytosol to vacuole then be transported from vacuole to cell wall through an unknown mechanism (Figure 1) [14-17].

Figure 1. Alternative models or pathways of monolignol transport in angiosperms and gymnosperms.

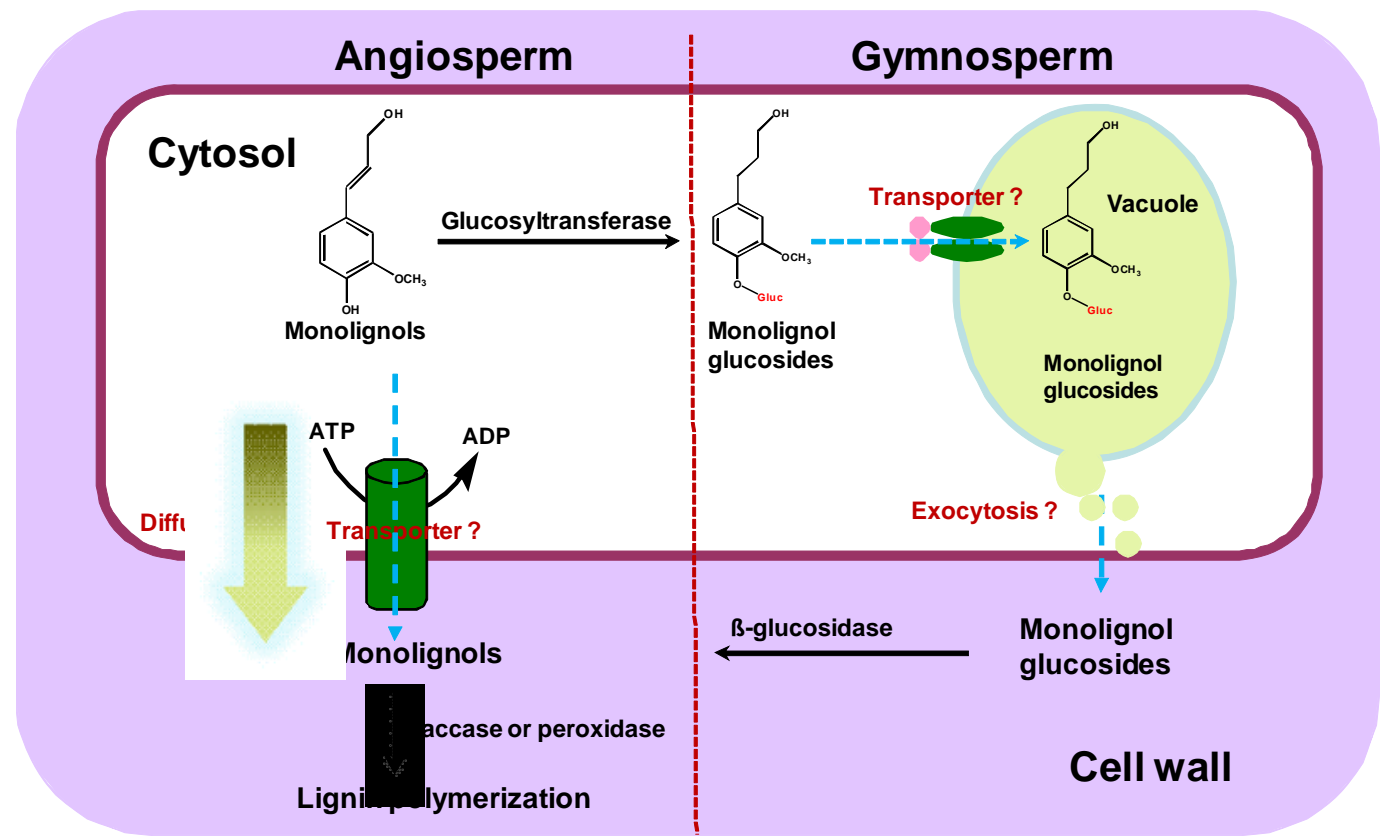

The symplastic monolignols may directly export to the cell wall through passive diffusion or by active transport. Alternatively, monolignols may be sequestrated as glucoconjugates into vacuole in gymnosperms. The stored glucosides may then be transported to the cell wall and hydrolyzed to the free monolignols for polymerization. 
Monolignol glycosylation is catalyzed by soluble UDP-glucose: coniferyl alcohol or sinapyl alcohol glucosyltransferase [18]. In a survey conducted by Ibrahim [19], coniferyl alcohol glucosyltransferase activity was detected in crude homogenates of all gymnosperms tested; among angiosperms, woody species exhibited higher enzyme activities than herbaceous species [19]. On the other hand, the specific $\beta$-glucosidases are proposed to release monolignols from the glucoconjugates in cell wall of differentiated xylem tissues. The corresponding enzymes have been characterized from both gymnosperm and angiosperm sources, such as spruce seedlings [20], chick pea cell suspension cultures and seedlings [21], soybean cell cultures, hypocotyls and roots [22], the differentiating xylem of Jack pine [23] and lodgepole pine [14]. Moreover, the apoplastic location of $\beta$-glucosidases was demonstrated by immunohistochemical techniques [24,25]. Consequently, the cDNAs encoding coniferin $\beta$-glucosidase have been isolated from both lodgepole pine (gymnosperm) and Arabidopsis (angiosperm). Their expression was proved highly specific in the site of lignifications [15,17]. Based on those evidences, a UDPG:coniferyl alcohol glucosyltransferase/coniferin $\beta$-glucosidase (CG) system was proposed to regulate the storage and mobilization of monolignols for lignin biosynthesis and plant lignification [14-17].

A small cluster of UDPG-glycosyltransferase genes were functionally characterized from Arabidopsis. The encoded enzymes were able to 4-O-glucosylate monolignols and other phenolics in vitro [26]. Down- or up-regulation of those identified glucosyltransferases resulted in the corresponding reduction or accumulation of the soluble monolignol glucosides in the transgenic roots or leaves of Arabidopsis [27]. However, no significant changes of lignin deposition was resulted from the disturbance of those glucosyltransferase genes' expression [3]. These data suggest that monolignol glucosylation might not play a role in exporting monolignols, at least in angiosperm species.

Moreover, a recent experiment feeding $\left[{ }^{3} \mathrm{H}\right]$-Phe to the dissected xylem of lodgepole pine showed that the radiolabel was incorporated directly into monolignols and to the lignin constituents accumulated in the cell wall. Neither substantial amount of radiolabel was associated with the glucoside of monolignol (coniferin), nor with the interior of the central vacuole, where coniferin is expectedly stored [28]. These data further argue against the role of monolignol glucosylation in the export of monolignols across the plasma membrane and imply that monolignol aglycone may be the chemical form for the transport.

Monolignols are relatively toxic, and unstable. Glucosylation of small molecule compounds is known to reduce their lipophilicity, thus preventing any further possibility of free diffusion across the lipid bilayer [29]. Therefore, the role of monolignol glucosylation may be to convert the highly active, unstable lignin precursors into the "storage form", as precursor reservoirs shielded them in a particular compartment.

\subsection{Mechanisms for the transport of monolignols across membranes}

The exact mechanism of monolignol transport from cytosol to cell wall remains unclear, although there are several proposed models or pathways. Early studies involving chemical analysis, radiotracers and microscopic examination suggested different mechanisms explaining the transport of monolignols (Figure 1). 


\subsubsection{Exocytosic transport via the Endoplasmic Reticulum- Golgi derived vesicles}

The non-cellulosic polysaccharides of plant cell wall, such as pectin and hemicelluloses, are well defined to be synthesized within the Golgi bodies and exported to the cell wall through an exocytosis mechanism [30]. Early autoradiographic, immunocytochemical, and ultrastructural studies suggested that transport of lignin precursors was also potentially via a similar ER-Golgi route as does the secretion of the wall matrix polysaccharides. A vesicle trafficking between cytosol and plasmalemma in differentiating tracheids of wheat xylem tissues was observed in the autoradiographic and ultrastructural studies [31]. Employing [ $\left.{ }^{3} \mathrm{H}\right]$-Phe, -tyrosine and -cinnamic acid to label the developing xylem, investigators found the radiolabels associated with the rough endoplasmic reticulum, the Golgi apparatus, and with some vesicles fused with the plasma membrane or aggregated in the cytoplasm near the bands of wall microtubules $[6,31,32]$. These findings suggest that the ER-Golgi apparatus is involved in the synthesis and transport of monolignols to the cell wall. Immunocytochemical localization of PAL and CAD in tracheary elements of Zinnia elegans also suggested that the immunolabels of PAL and CAD seemed not only to disperse in the cytoplasmic matrix, but also localize to the Golgi-derived vesicles and the developing secondary thickenings [7]. These early observations led to the assumption that monolignols as the content of Golgi-derived vesicles could be exported to the cell wall by fusion of vesicular-membrane with the plasmalemma. However, in those early autoradiographic studies, most of the radioactive intermediates of monolignols, might be extracted during the exchange of organic solvents for embedding the specimens in resin. Moreover, the radioactive lignin precursors such as Phe are assimilated efficiently to both lignin and proteins. These could interferer the interpretation of autoradiography. In a recent study, Kaneda et al. [28] adopted cryofixation and freezing substitution to prepare the labeled lodgepole pine xylem cells. These new cytological techniques substantially minimized the damage on the sectioned cells, thus preventing pseudo-autographic images. Using the $\left[{ }^{3} \mathrm{H}\right]$-Phe radiotracer to feed the dissected developing xylem tissue of the lodgepole pine, together with the selective inhibition of phenylpropanoid and protein biosynthesis, respectively, they discovered that the Phe-radiolabel in ER-Golgi was primarily incorporated into proteins, not monolignols. Further, they found that the abundant Golgi and Golgivesicle clusters that were observed in the developing xylem cells did not load with phenylpropanoids. Therefore, these up-to-date autoradiographic results suggest that ER-Golgi-vesicle mediated exocytosis is unlikely to play a major role in monolignol transport.

\subsubsection{Passive diffusion}

Genetic engineering and chemical compositional analyses revealed that lignin biosynthesis displays considerable plasticity. Besides three classical monolignols ( $p$-coumaryl, coniferyl, and sinapyl alcohols), several other phenolic components are also incorporated into lignin polymer. These include the products from the incomplete monolignol biosynthesis, such as 5-hydroxyconiferyl alcohol [1], hydroxycinnamaldehydes [33] and hydroxycinnamic acids [34,35], as well as the enzymatic derivatives of monolignols, such as sinapyl $p$-hydroxybenzoate, coniferyl and sinapyl $p$-coumarate [36], and coniferyl and sinapyl acetate [37,38]. Such accommodation of alternative monomers in ligninifcation infers a potential non-specific passive diffusion of lignin precursors across the plasma 
membrane [3]. This passive diffusion notion was further evident from the in vitro partitioning experiments by using immobilized liposomes or lipid bilayer dises as the model membranes. When lignin precursor analogs were incubated with liposomes or lipid bilayer discs, they were observed to be easily partitioned into the artificial membrane phase [39,40]. However, there are a variety of phenolics synthesized in the cytosol of a living cell, and all have a similar hydrophobic nucleus (phenyl ring), it is not clear how monolignols and/or their particular derivatives are selected and diffuse across the plasma membranes.

\subsubsection{Transporter-mediated monolignol sequestration and export}

Many studies show that lignin monomers are differentially deposited in discrete regions of various types of lignifying cell wall. For example, lignin in the vessel cell walls of birch wood is derived mainly from coniferyl alcohol, whereas the fiber wall incorporates both sinapyl and coniferyl alcohols [41]. Similarly, in Arabidopsis stems, the lignin of the vascular bundle rich in vessels primarily contains $\mathrm{G}$ units, while the interfascicular fibers are enriched in S units [42]. In spruce wood, the lignin of the middle lamella embeds more $p$-coumaryl alcohol units than does the secondary wall lignin that was mainly derived from coniferyl alcohol [43]. When feeding the labeled monolignols into developing xylem, the radiolabeled p-coumaryl alcohol was preferentially deposited in the middle lamella/cell corners, whereas coniferyl alcohol was mainly located in the secondary wall [44]. Moreover, during development of secondary cell wall to form S1-, S2-, and S3- layers, the three kinds of monolignols exhibited sequential deposition, in the order of p-coumaryl alcohol (for $\mathrm{H}$ unit), coniferyl alcohol (G unit) and sinapyl alcohol (S unit) [44-46]. These data suggest that lignin monomer deposition into cell wall is a highly organized, regulated process, and that some active transport mechanisms may selectively permit the deposition of the particular monolignols.

Recent functional genomic, global transcriptomic and proteomic studies in both gymnosperms and angiosperms frequently uncovered some putative membrane transporters potentially involved in xylem differentiation, and secondary cell-wall formation. They are highly expressed in the lignified wood tissues. For example, a number of ESTs encoding for ATP-binding cassette (ABC) transporters were disclosed from several studies on gene expression during wood transformation [47-50]. When analyzing the data of the first generation of loblolly pine microarray, the distinct ABC transporter-like genes were found abundant in the mature wood and compression wood, wherein different types of lignins accumulated $[50,51]$. Similarly, a set of $A B C$ transporters were identified in a microarray study of gene expression during lignification of Arabidopsis stem. Those ABC transporters were coordinately expressed with the known lignin biosynthetic enzymes $[52,53]$. In a recent proteomics study on plasma membrane from poplar leaf, xylem and cambium/phloem tissues, a set of $\mathrm{ABC}$ transporters showed a rather "specific" tissue distribution [54]. One member in the subfamily B and three in $\mathrm{G}$ were identified in cambium/phloem only; while a member of subfamily B was specific to xylem, and its Arabidopsis homolog, ABCB15 (MDR13), was correlated well with lignin biosynthetic genes in a transcritpomic studies [52]. These data imply that membrane transporters, particularly, ABC transporters, might have a significant role in cell wall lignification, presumably in monolignol transport to the cell wall. 


\subsection{Membrane transporters and their implication in phenolic transport}

Sequestering small molecule metabolites within particular intracellular compartments or transferring them between different cell types require specific multidrug transporters. The multidrug transporters form a large class of membrane proteins present in the cells of most organisms. These transporters fall into five families: The ATP-binding cassette superfamily (ABC), the major facilitator superfamily, the small multidrug resistance family, the resistance-nodulation-cell division family, and the multidrug and toxic compound extrusion (MATE) family [55].

The $\mathrm{ABC}$ transporters constitute a large, diverse, and ubiquitous superfamily. The majority of $\mathrm{ABC}$ genes encode membrane-bound proteins that participate in transporting a wide range of molecules across membranes [56-58]. The ABC proteins can be broadly categorized as importers or exporters, depending on the direction of movement relative to the cytoplasm [59]. According to a recent inventory and new nomenclature for $\mathrm{ABC}$ proteins, they can be divided into nine subfamilies, namely ABCA-I, with all subfamilies except $\mathrm{H}$ found in plants [58]. ABC proteins are defined by their possession of an ATP-binding cassette, also known as the nucleotide-binding domain (NBD) [58,60]. In addition to NBDs, $\mathrm{ABC}$ transporters also encompass transmembrane domains (TMDs), each composed of several hydrophobic $\alpha$-helices. The core units comprising a functional ABC transporter consist of four domains: Two NBDs, and two TMDs. The former cooperatively bind and hydrolyze ATP, providing the driving force for transport, and the latter are involved in substrate recognition and translocation across the lipid bilayer. Although most prokaryotic ABC proteins are encoded as the separate TMD and NBD subunits or as "one-half-size" (one TMD and one NBD) transporters, in the majority of eukaryotic ABC transporters, all four domains (two TMDs and two NBDs) are contiguous on a single polypeptide, constituting a full length transporter in the "forward" (TMD1-NBD1-TMD2NBD2) orientation, or the "reverse" (NBD1-TMD1-NBD2-TMD2) orientation. In a particular subfamily, the ABCC (MRP), the arrangement is in the forward orientation, but some proteins, e.g., MRP1 have extra five $\alpha$ helices (sometimes designated as TMD0) at the $\mathrm{N}$ terminus [59,61].

The Arabidopsis and rice (Oryza sativa) genomes each contains more than 120 putative ABC transporters, but only a few have been characterized [62]. These proteins show diverse transport activities to a variety of small molecule metabolites, such as glutathione-conjugates [63,64], chlorophyll catabolites [63,64], peptides [65], lipids [66], auxins [67-69], abscisic acid [70,71], inorganic ions [72], malate [73], wax and cutin precursors [74,75], defensive secondary metabolites such as antifungal terpenoids [76], tropane alkaloids (hyoscyamine and scopolamine) [77], benzylisoquinoline alkaloid (berberine) [78,79], and xenobiotics [80].

Among these identified $\mathrm{ABC}$ transporters, a few are revealed responsible for phenolic/polyphenolic transport [81]. A membrane-potential dependent ABC-like transporter was demonstrated to mediate the vacuolar uptake of flavone glucuronides in rye [82,83]. Genetic suppression of the maize tonoplastlocalized GS-X pump, the ZmMRP3, resulted in the reduced anthocyanin accumulation [84], although the transport activity of ZmMRP3 to anthocyanin has yet to be shown. In addition, plasma membrane $\mathrm{ABC}$ transporter-mediated secretion of the isoflavone genistein in soybean roots has been reported [85], but the gene encoding the protein is yet to be identified.

Besides involvement of the energized primary transporter, i.e., $\mathrm{ABC}$ transporters, transporting or sequestering plant small molecule metabolites is also mediated by active secondary transport systems 
[86]. The MATE family is the most recently classified multidrug resistance-conferring, secondary transporter family [55]. These proteins use an electrochemical gradient (usually $\mathrm{H}^{+} / \mathrm{Na}^{+}$) as the driving force to transport other substrates across membranes. Their function and activity largely depend on various types of $\mathrm{H}^{+}$-ATPases [81]. The MATE family is divided into three groups based on sequence relatedness. Prokaryotes have members of the two more related groups, whereas the third group is composed exclusively of proteins from eukaryotes. Members of this third group of MATE proteins are encoded by about 56 genes in the Arabidopsis genome [87]. These genes often are organized in tandem repeats in the genome.

Biochemical analyses of plant MATE family members suggest these proteins function as efflux pumps. Several MATE transporters have been characterized. These include Arabidopsis ALF5 that exports toxic cations such as tetramethylammonium thus protecting roots from exogenous inhibitory compounds [88], AtDTX1 that confers the efflux of toxic compounds norfloxacin, berberine and cadmium tolerance when expressed in Escherichia coli [89], and the Arabidopsis MATE transporter TT12 that acts as a cyanidin-3-O-glucoside/ $\mathrm{H}^{+}$-antiporter mediates vacuolar sequestration of anthocyanins [90,91]. When expressed in yeast, TT12 and its ortholog MATE1 from M. truncatula preferentially transport epicatechin 3'-O-glucoside, the proposed precursor for the synthesis of proanthocyanidins [92]. Therefore, TT12 and its homologs are also responsible for the accumulation of proanthocyanidins in Arabidopsis and legume seed coats. Recently, two MATE-type transporters from grapevine are characterized as vacuolar transporters of acylated anthocyanins [93]. In addition, a tobacco MATE homolog is demonstrated responsible for vacuolar sequestration of nicotine alkaloid in the aerial parts of tobacco $[94,95]$.

Although both the ABC and MATE membrane-transporters are reportedly involved in the vacuolar sequestration or transport of a range of phenolic glycosides and glutathione conjugates, the transport activity of two types of membrane transporters to small molecule compounds in plant cells displays disparate characteristics [87,96]. Collectively: 1) different glucoconjugates are moved by different transporters using distinct mechanisms. For instance, a flavonoid glucoside, isovitexin, a native Cglucoside in barley, was conveyed into the isolated vacuoles of barley via electrochemical gradientdependent secondary transport. In contrast, the herbicide glucoside of hydroxyprimisulfuron was taken up by the directly energized primary transport mechanism [97]. 2) Identical compounds under distinct circumstances (e.g., intrinsic or heterologous) in cells may be transported by different mechanisms. The uptake of the main barley flavonoid saponarin, an apigenin glucoside, into barley vacuoles occurred via a proton pump, whereas the transport of the same compound into Arabidopsis vacuoles, a heterologous plant that does not generate this metabolite, displayed typical characteristics of an $\mathrm{ABC}$ transporter-mediated process [83]. Moreover, the conveyance of structurally similar endogenous metabolites also necessitates distinct transporters. Transport of anthocyanin into vacuoles in maize involved $\mathrm{ABC}$ transporter MRPs [84]. However, sequestration of proanthocyanidins, a group of polyphenolics structurally related to anthocyanidins, requires $\mathrm{H}^{+}$-gradient dependent MATE transport [92]. The Arabidopsis mutant transparent testa12 (tt12), lacking the gene encoding a MATE family secondary transporter-like protein exhibited strongly reduced proanthocyanidine deposition in the vacuoles of endothelial cells [90,91]. 3) Transport of phenolics is affected by the presence of the hydrophilic ligand (e.g., glucose or glutathione). The uptake of phenolic glucosides of $p$-hydroxycinnamic acid and $p$-hydroxybenzoic acid by vacuolar vesicles purified from red beet (Beta vulgaris) 
seemingly was via an $\mathrm{H}^{+}$- gradient-dependent transport mechanism [98]. In contrast, when cinnamic acid was conjugated with glutathione, it was transported into the vesicles via a GS-X pump, defined as ABCC protein [99]. These data imply that the sugar moiety may be a preferred 'tag' recognized by the secondary transporters, whereas the glutathione moiety is preferred by MRP (ABCC) proteins $[100,101]$ and 4) a given phytochemical can be transported by more than one membrane transporters, on the other hand, a transporter can involve in the secretion of more than one secondary metabolite [102].

\subsection{ABC-like transporters are involved in the transport of monolignols and their glucosides across membranes}

Although several pieces of information suggest the potential involvement of membrane transporters in the sequestration and transport of lignin precursor across membranes, the direct biochemical evidence has been missing. To clarify whether the transport of lignin precursor is the membrane transporter-mediated active process, exploration was recently conducted in authors' group by the in vitro uptake assay that incubates the isolated plasma and vacuolar membrane vesicles from both Arabidopsis young rosette leaves and the roots of poplar with monolignols and/or their glucosides [103]. Our studies demonstrate that the transport of lignin precursors across both plasma and vacuolar membranes is largely dependent on the presence of ATP. In the absence of ATP, the transport activity of either plasma or vacuolar membrane vesicles to monolignols or their glucosides is severely impaired, only $20 \sim 30 \%$ of that detected in the presence of ATP. Among different nucleotide triphosphates, ATP mostly promotes the transport activity. The specific ABC-type transporter inhibitors largely reduce the transport activity of plasma or vacuolar membrane vesicles to lignin precursors. Furthermore, in the presence of ATP, the vacuolar membrane vesicles prepared from Arabidopsis rosette leaves display considerable activity in sequestering the glucoconjugates coniferin and syringin, but a very limited activity to monolignol aglycones. In contrast, the plasma membrane vesicles are inactive to the glucoconjugated monolignols and prefer for the aglycones (Figure 2). These data suggest that glucosylation of monolignols is a prerequisite for their vacuolar sequestration while the aglycones are required for the direct transport into cell walls of Arabidopsis. The current studies complement previous genetic observation, wherein down- or up-regulating the expression of Arabidopsis UDPG: monolignol glucosyltransferases entailed the corresponding reduction or accumulation of the soluble monolignol glucosides in transgenic roots or leaves [27], but a change in lignin content or composition was not observed.

The different chemical forms of monolignols required in the ATP-dependent sequestration and transport also implicates the distinct classes of $\mathrm{ABC}$ transporters involved in diverting and partitioning the polarized "storage form" glucosides of monolignols into vacuoles, and the hydrophobic aglycones across plasmalemma. In the assay, we also observed that compared to vacuolar vesicles, plasma membranes displayed notable promiscuity in conveying different phenolics in the presence or absence of ATP molecules. The less selectivity of plasma membranes might explain the observed plasticity of lignin biosynthesis. The promiscuous active transport and/or the low level of intrinsic plasmalemma diffusion may lead to the deposition of non-classic lignin precursors into the cell wall. 
Figure 2. ATP-dependent, selective uptake of monolignols or their glucosides by vacuolar vesicles and the inside-out plasma membrane vesicles.

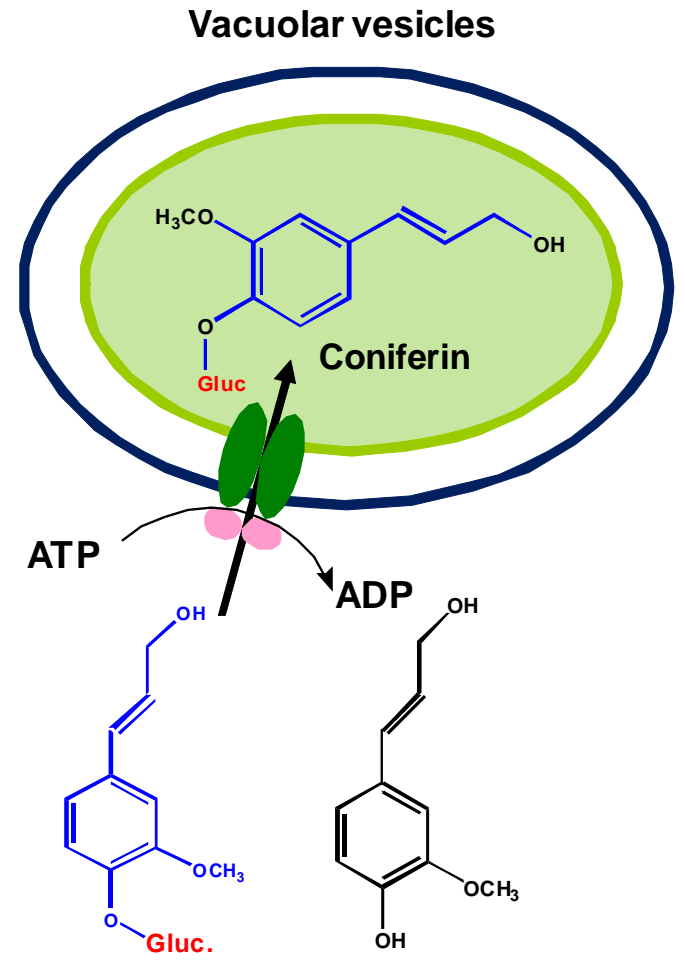

Coniferin

Coniferyl alcohol

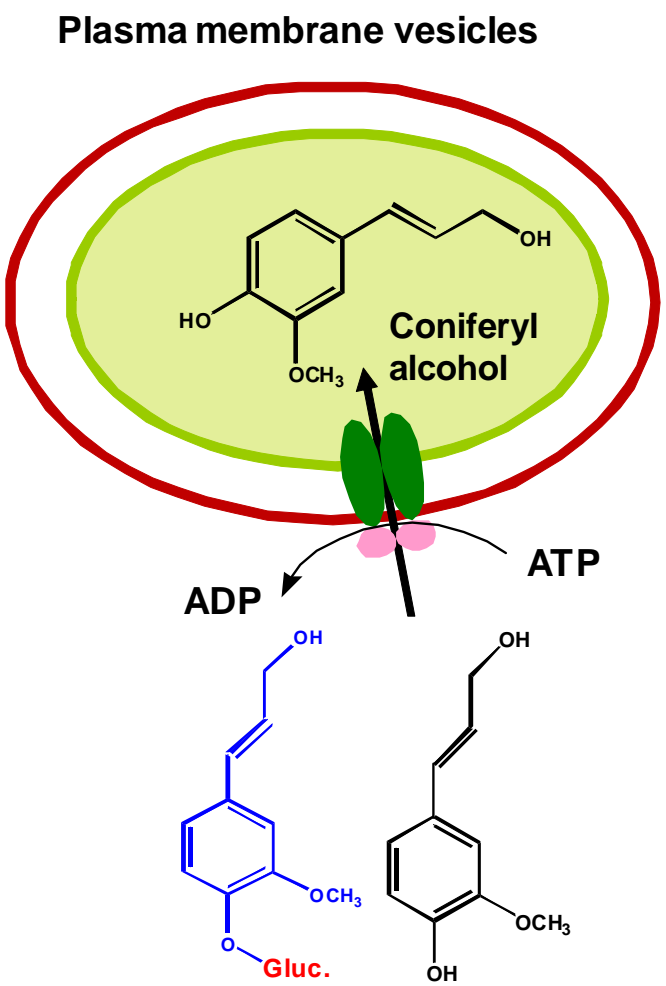

Coniferin Coniferyl alcohol

Although $\mathrm{ABC}$ transporter inhibitors severely disrupt the uptake of plasma and vacuolar membrane vesicles to monolignols or their glucosylated derivatives in the uptake assays, ionophoric agents, such as gramicidin $\mathrm{D}$, nigericin and $\mathrm{NH}_{4} \mathrm{Cl}$ that disturb the activities of MATE family transporters, showed less inhibitory effect on the uptake of lignin precursors. After ionophore treatments, both plasma and vacuolar membrane vesicles remained $60 \sim 80 \%$ of the transport activity in the presence of ATP. These data imply that the secondary energized MATE transporters may not play a vital role as the ABC-type transporters do in mediating lignin precursor transport. This observation is also consistent with those transcriptomics and proteomics studies on cell wall formation. Analyzing global gene expression and examining the integral plasma membrane proteins in differentiated xylem tissues did not reveal particular MATE transporters correlated with lignin biosynthesis [52,54].

\section{Conclusions and Perspectives}

Transport of lignin monomers across cell membranes is the critical, albeit least investigated, step in plant lignification. The up- to-date information indicates that: (1) glycoconjugates of monolignols may not be the required transport form across the plasma membranes, although lignin monomeric precursors in all gymnosperms and a few angiosperms are often glucosylated. Like many other stored phenolics, glucosylation of monolignols may simply be to facilitate the vacuolar sequestration of those active compounds for storage. At least in angiosperms (both the tested herbaceous and woody plants), monolignol aglycones are directly exported across plasma membranes into the cell wall. Whether there is disparate mechanism for sequestering and transporting lignin precursors in gymnosperms remains to 
be further clarified. (2) Vacuolar sequestration and plasma membrane transport of lignin precursors require membrane protein-mediated active transport mechanisms. Although, the possibility of a small portion of passive diffusion can't be excluded and more than one mechanism may be involved in the transport of monolignols and their glucosides, $\mathrm{ABC}$-like transporters are evidenced as the major player. In future, the particular ABC-like transporter proteins need to be identified.

Understanding the basis for the sequestration and transport of lignin monomers and identifying the molecular components involved in those processes are significantly meaningful for manipulating plant lignification, therefore improving the digestibility of cell wall biomass. Three potential applications/consequences of the studies can be envisioned.

(1) Elucidating the vacuolar sequestration mechanism of lignin precursors and isolating the related vacuolar transporters will enable a potential genetic engineering strategy to selectively divert lignin precursors into the "chemical sink" within the cells and reduce the amount of lignin precursors deposited into cell wall for polymerization, by which to reduce cell wall recalcitrance, meanwhile, maintain or promote the organic carbon storage in particular cell compartment (e.g., vacuole).

(2) Similarly, exploring the mechanisms for monolignol export across plasma membrane and subsequently characterizing the potential biochemical factors involved may lead to a rational solution for controlling the deposition of lignin precursors, thus precisely manipulating both lignin content and composition in the targeted cell wall. This will enable us to alter the ratio of the labile to condensed lignin to produce more easily cleavable biopolymers.

(3) Plant membrane transporters are not only involved in sequestering and transporting lignin precursors, but also contribute to many other biological processes in wood formation, e.g., hormone transportation and defense responses to environmental stresses. In the future, systematically identifying and characterizing the membrane-transporters involved in plant lignification will benefit both the trials to tailor lignin biosynthesis and the sustainable production of cell wall biomass.

\section{Acknowledgements}

This work was supported by the Division of Chemical Sciences, Geosciences, and Biosciences, Office of Basic Energy Sciences of the U.S. Department of Energy through Grant DEAC0298CH10886 to C.J.L., by the Laboratory Directed Research and Development program (LDRD No. 11-007) of Brookhaven National Laboratory, contracted with U.S. Department of Energy, and by the Division of Molecular and Cellular Biosciences of National Science Foundation.

\section{References and Notes}

1. Boerjan, W.; Ralph, J.; Baucher, M. Lignin biosynthesis. Annu. Rev. Plant Biol. 2003, 54, 519-546.

2. Ralph, J.; Lundquist, K.; Brunow, G.; Lu, F.; Kim, H.; Schatz, P.F.; Marita, J.M.; Hatfield, R.D.; Christensen, J.H.; Boerjan, W. Lignins: natural polymers from oxidative coupling of 4-hydroxyphenylpropanoids. Phytochem. Rev. 2004, 3, 29-60.

3. Vanholme, R.; Morreel, K.; Ralph, J.; Boerjan, W. Lignin engineering. Curr. Opin. Plant Biol. 2008, 11, 278-285.

4. Li, X.; Weng, J.K.; Chapple, C. Improvement of biomass through lignin modification. Plant J. 2008, 54, 569-581. 
5. Chen, F.; Dixon, R.A. Lignin modification improves fermentable sugar yields for biofuel production. Nat. Biotechnol. 2007, 25, 759-761.

6. Takabe, K.; Fujita, M.; Harada, H.; Saiki, H. Autoradiographic investigations of lignification in the cell walls of cryptomeria (Cryptomeria japonica d. Don). Mokuzai Gakkaishi 1985, 31, 613-619.

7. Nakashima, J.; Mizuno, T.; Takabe, K.; Fujita, M.; Saiki, H. Direct visualization of lignifying secondary wall thickenings in Zinna elegans cells in culture. Plant Cell Physiol. 1997, 38, 818-827.

8. Achnine, L.; Blancaflor, E.B.; Rasmussen, S.; Dixon, R.A. Colocalization of L-phenylalanine ammonia-lyase and cinnamate 4-hydroxylase for metabolic channeling in phenylpropanoid biosynthesis. Plant Cell 2004, 16, 3098-3109.

9. Whetten, R.; Sederoff, R. Lignin biosynthesis. Plant Cell 1995, 7, 1001-1013.

10. Terazawa, M.; Miyake, M. Phenolic compounds in living tissue of woods. II. Seasonal variations of phenolic glycosides in the cambial sap of woods. Mokuzai Gakkaishi 1984, 30, 329-334.

11. Leinhos, V.; Savidge, R.A. 1solation of protoplasts from developing xylem of Pinus banksiana and Pinus strobos. Can. J. For. Res. 1993, 23, 343-348.

12. Savidge, R.A. Coniferin, a biochemical indicator of commitment to tracheid differentiation in conifers. Can. J. Bot. 1989, 67, 2663-2668.

13. Savidge, R.A. A biochemical indicator of commitment to tracheid differentiation in Pinus contorta. Can. J. Bot. 1988, 66, 2009-2012.

14. Dharmawardhana, D.P.; Ellis, B.E.; Carlson, J.E. A $\beta$-glucosidase from lodgepole pine xylem specific for the lignin precursor coniferin. Plant Physiol. 1995, 107, 331-339.

15. Dharmawardhana, D.P.; Ellis, B.E.; Carlson, J.E. cDNA cloning and heterologous expression of coniferin $\beta$-glucosidase. Plant Mol. Biol. 1999, 40, 365-372.

16. Samuels, A.L.; Rensing, K.H.; Douglas, C.J.; Mansfield, S.D.; Dharmawardhana, D.P.; Ellis, B.E. Cellular machinery of wood production: Differentiation of secondary xylem in Pinus contorta var. Iatifolia. Planta 2002, 216, 72-82.

17. Escamilla-Treviñ, L.L.; Chen, W.; Card, M.L.; Shih, M.-C.; Cheng, C.-L.; Poulton, J.E. Arabidopsis thaliana $\beta$-glucosidases BGLU45 and BGLU46 hydrolyse monolignol glucosides. Phytochemistry 2006, 67, 1651-1660.

18. Steeves, C.; F“orster, H.; Pommer, U.; Savidge, R. Coniferyl alcohol metabolism in conifers. I. Glucosidic turnover of cinnamyl aldehydes by UDPG: Coniferyl alcohol glucosyltransferase from pine cambium. Phytochemistry 2001, 57, 1085-1093.

19. Ibrahim, R.K. Glucosylation of lignin precursors by uridine diphosphate glucose: Coniferyl alcohol glucosyltransferase in higher plants. Z. Pflanzenphysiol. 1977, 85, 253-262.

20. Marcinowski, S.; Grisebach, H. Enzymology of lignification. Cell wall-bound $\beta$-glucosidase from coniferin from spruce (Picea abies) seedlings. Eur. J. Biochem. 1978, 87, 37-44.

21. Hösel, W.; Surholt, E.; Borgmann, E. Characterization of beta-glucosidase isoenzymes possibly involved in lignification from chick pea (Cicer arietinum L.) cell suspension cultures. Eur. J. Biochem. 1978, 84, 487-492.

22. Hösel, W.; Todenhagen, R. Characterization of a beta-glucosidase from Glycine max which hydrolyses coniferin and syringin. Phytochemistry 1980, 19, 1349-1353. 
23. Leinhos, V.; Udagama-Randeniya, P.V.; Savage, R.A. Purification of an acidic coniferinhydrolysing glucosidase from developing xylem of Pinus banksiana. Phytochem. 1994, 37, 311-315.

24. Marcinowski, S.; Falk, H.; Hammer, D.K.; Hoyer, B.; Grisebach, H. Appearance and localization of a beta-glucosidase hydrolyzing coniferin in spruce (Picea abies) seedlings. Planta 1979, 144, 161-165.

25. Burmeister, G.; Hosel, W. Immunohistochemical localization of beta-glucosidase in lignin and isoflavone metabolism in Cicer arietinum L. seedlings. Planta 1981, 152, 578-586.

26. Lim, E.K.; Li, Y.; Parr, A.; Jackson, R.; Ashford, D.A.; Bowles, D.J. Identification of glucosyltransferase genes involved in sinapate metabolism and lignin synthesis in Arabidopsis. $J$. Biol. Chem. 2001, 276, 4344-4349.

27. Lanot, A.; Hodge, D.; Jackson, R.G.; George, G.L.; Elias, L.; Lim, E.-K.; Vaistij, F.E.; Bowles, D.J. The glucosyltransferase UGT72E2 is responsible for monolignol 4-O-glucoside production in Arabidopsis thaliana. Plant J. 2006, 48, 286-295.

28. Kaneda, M.; Rensing, K.H.; Wong, J.C.; Banno, B.; Mansfield, S.D.; Samuels, A.L. Tracking monolignols during wood development in lodgepole pine. Plant Physiol. 2008, 147, 1750-1760.

29. Bowles, D.; Lim, E.-K.; Poppenberger, B.; Vaistij, F.V. Glycosyltransferases of lipophilic small molecules. Annu. Rev. Plant Biol. 2006, 57, 567-597.

30. Lerouxel, O.; Cavalier, D.M.; Liepman, A.H.; Keegstra, K. Biosynthesis of plant cell wall polysaccharides- a complex process. Curr. Opin. Plant Biol. 2006, 9, 621-630.

31. Pickett-Heaps, J.D. Xylem wall deposition: Radioautographic investigations using lignin precursors. Protoplasma 1968, 65, 181-205.

32. Fujita, M.; Harada, H. Autoradiographic investigations of cell wall development. II. Tritiated phenylalanine and ferulic acid assimilation in relation to lignification. Mokuzai Gakkaishi 1979, 25, 89-94.

33. Morreel, K.; Ralph, J.; Kim, H.; Lu, F.; Goeminne, G.; Ralph, S.; Messens, E.; Boerjan, W. Profiling of oligolignols reveals monolignol coupling conditions in lignifying poplar Xylem. Plant Physiol. 2004, 136, 3537-3549.

34. Leple, J.C.; Dauwe, R.; Morreel, K.; Storme, V.; Lapierre, C.; Pollet, B.; Naumann, A.; Kang, K.Y.; Kim, H.; Ruel, K.; Lefebvre, A.; Joseleau, J.P.; Grima-Pettenati, J.; De Rycke, R.; Andersson-Gunneras, S.; Erban, A.; Fehrle, I.; Petit-Conil, M.; Kopka, J.; Polle, A.; Messens, E.; Sundberg, B.; Mansfield, S.D.; Ralph, J.; Pilate, G.; Boerjan, W. Downregulation of cinnamoylcoenzyme A reductase in poplar: multiple-level phenotyping reveals effects on cell wall polymer metabolism and structure. Plant Cell 2007, 19, 3669-3691.

35. Ralph, J.; Kim, H.; Lu, F.; Grabber, J.H.; Leple, J.C.; Berrio-Sierra, J.; Derikvand, M.M.; Jouanin, L.; Boerjan, W.; Lapierre, C. Identification of the structure and origin of a thioacidolysis marker compound for ferulic acid incorporation into angiosperm lignins (and an indicator for cinnamoyl CoA reductase deficiency). Plant J. 2008, 53, 368-379.

36. Lu, F.; Ralph, J.; Morreel, K.; Messens, E.; Boerjan, W. Preparation and relevance of a crosscoupling product between sinapyl alcohol and sinapyl p-hydroxybenzoate. Org. Biomol. Chem. 2004, 2, 2888-2890. 
37. Del Rio, J.C.; Marques, G.; Rencoret, J.; Martinez, A.T.; Gutierrez, A. Occurrence of naturally acetylated lignin units. J. Agric. Food. Chem. 2007, 55, 5461-5468.

38. del Rio, J.C.; Rencoret, J.; Marques, G.; Gutierrez, A.; Ibarra, D.; Santos, J.I.; Jimenez-Barbero, J.; Zhang, L.; Martinez, A.T. Highly acylated (acetylated and/or p-coumaroylated) native lignins from diverse herbaceous plants. J. Agric. Food Chem. 2008, 56, 9525-9534.

39. Boija, E.; Lundquist, A.; Edwards, K.; Johansson, G. Evaluation of bilayer disks as plant cell membrane models in partition studies. Anal. Biochem. 2007, 364, 145-152.

40. Boija, E.; Lundquist, A.; Nilsson, M.; Edwards, K.; Isaksson, R.; Johansson, G. Bilayer disk capillary electrophoresis: A novel method to study drug partitioning into membranes. Electrophoresis 2008, 29, 3377-3383.

41. Fergus, B.J.; Goring, D.A.I. The distribution of lignin in birch wood as determined by ultraviolet microscopy. Holzforschung 1970, 24, 118-124.

42. Chapple, C.; Vogt, T.; Ellis, B.E.; Somerville, C. Arabidopsis mutant defective in phenylpropanoid pathway. Plant Cell 1994, 4, 1413-1424.

43. Whiting, P.; Goring, D.A.I. Chemical characterization of tissue fractions from the middle lamella and secondary wall of black spruce tracheids. Wood Sci. Technol. 1982, 16, 261-267.

44. Terashima, N.; Fukushima, K.; He, L.-F.; Takabe, K. Comprehensive model of the lignified plant cell wall. In Forage Cell Wall Structure and Digestibility; Jung, H.G., Buxton, D.R., Hatfield, R.D., Ralph, J., Eds.; ASA-CSSA-SSSA: Madison, WI, USA, 1993; pp. 247-270.

45. Terashima, N.; Fukushima, K.; Tsuchiya, S. Heterogeneity in formation of lignin VII. An autoradiographic study on the formation of guaiacyl and syringyl lignin in poplar. J. Wood Chem. Technol. 1986, 6, 495-504.

46. Terashima, N.; Fukushima, K.; Takabe, K. Heterogeneity in formation of lignin VIII. An autoradiographic study on the formation of guaiacyl and syringyl lignin in Magnolia kobus dc. Holzforschung 1986, 40, 101-105.

47. Allona, I.; Quinn, M.; Shoop, E.; Swope, K.; Cyr, S.S.; Carlis, J.; Riedl, J.; Retzel, E.; Campbell, M.M.; Sederoff, R.E.A. Analysis of xylem formation in pine by cDNA sequencing. Proc. Natl. Acad. Sci. USA 1998, 95, 9693-9698.

48. Hertzberg, M.; Aspeborg, H.; Schrader, J.; Andersson, A.; Erlandsson, R.; Blomqvist, K.; Bhalerao, R.; Uhlen, M.; Teeri, T.T.; Lundeberg, J.; Sundberg, B.; Nilsson, P.; Sandberg, G. A transcriptional roadmap to wood formation. Proc. Natl. Acad. Sci. USA 2001, 98, 14732-14737.

49. Kirst, M.; Johnson, A.F.; Baucom, C.; Ulrich, E.; Hubbard, K.; Staggs, R.; Paule, C.; Retzel, E.; Whetten, R.; Sederoff, R. Apparent homology of expressed genes from wood-forming tissues of loblolly pine (Pinus taeda) with Arabidopsis thaliana. Proc. Natl. Acad. Sci. USA 2003, 100, 7383-7388.

50. Egertsdotter, U.; van Zyl, L.M.; MacKay, J.; Peter, G.; Kirst, M.; Clark, C.; Whetten, R.; Sederoff, R. Gene expression during formation of earlywood and latewood in loblolly pine: expression profiles of 350 genes. Plant Biol. (Stuttg) 2004, 6, 654-663.

51. Whetten, R.; Sun, Y.H.; Zhang, Y.; Sederoff, R. Functional genomics and cell wall biosynthesis in loblolly pine. Plant Mol. Biol. 2001, 47, 275-291.

52. Ehlting, J.; Mattheus, N.; Aeschliman, D.S.; Li, E.; Hamberger, B.; Cullis, I.F.; Zhuang, J.; Kaneda, M.; Mansfield, S.D.; Samuels, L.; Ritland, K.; Ellis, B.E.; Bohlmann, J.; Douglas, C.J. 
Global transcript profiling of primary stems from Arabidopsis thaliana identifies candidate genes for missing links in lignin biosynthesis and transcriptional regulators of fiber differentiation. Plant J. 2005, 42, 618-640.

53. Douglas, C.J.; Ehlting, J. Arabidopsis thaliana full genome longmer microarrays: a powerful gene discovery tool for agriculture and forestry. Transgenic Res. 2005, 14, 551-561.

54. Nilsson, R.; Bernfur, K.; Gustavsson, N.; Bygdell, J.; Wingsle, G.; Larsson, C. Proteomics of plasma membranes from poplar trees reveals tissue distribution of transporters, receptors, and proteins in cell wall formation. Mol. Cell Proteomics 2010, 9, 368-387.

55. Brown, M.H.; Paulsen, I.T.; Skurray, R.A. The multidrug efflux protein NorM is a prototype of a new family of transporters. Mol. Microbiol. 1999, 31, 393-395.

56. Higgins, C.F. ABC transporters: From microorganisms to man. Annu. Rev. Cell Biol. 1992, 8, 67-113.

57. Sa'nchez-Ferna'ndez, R.; Davies, E.T.G.; Coleman, J.O.D.; Rea, P.A. The Arabidopsis thaliana ABC protein superfamily: a complete inventory. J. Biol. Chem. 2001, 276, 30231-30244.

58. Verrier, P.J.; Bird, D.; Burla, B.; Dassa, E.; Forestier, C.; Geisler, M.; Klein, M.; Kolukisaoglu, U.; Lee, Y.; Martinoia, E.; Murphy, A.; Rea, P.A.; Samuels, L.; Schulz, B.; Spalding, E.J.; Yazaki, K.; Theodoulou, F.L. Plant ABC proteins - a unified nomenclature and updated inventory. Trends Plant Sci. 2008, 13, 151-159.

59. Rea, P.A. Plant ATP-Binding Cassette transporters. Annu. Rev. Plant Biol. 2007, 58, 347-375.

60. Higgins, C.F.; Linton, K.J. The ATP switch model for ABC transporters. Nat. Struct. Mol. Biol. 2004, 11, 918-926.

61. Schulza, B.; Kolukisaoglu, H.U. Genomics of plant ABC transporters: The alphabet of photosynthetic life forms or just holes in membranes? FEBS Letters 2006, 580, 1010-1016.

62. Jasinski, M.; Ducos, E.; Martinoia, E.; Boutry, M. The ATP-binding cassette transporters: Structure, function, and gene family comparison between rice and arabidopsis. Plant Physiol. 2003, 131, 1169-1177.

63. Lu, Y.P.; Li, Z.S.; Drozdowicz, Y.M.; Hortensteiner, S.; Martinoia, E.; Rea, P.A. AtMRP2, an Arabidopsis ATP binding cassette transporter able to transport glutathione S-conjugates and chlorophyll catabolites: functional comparisons with Atmrp1. Plant Cell 1998, 10, 267-282.

64. Tommasini, R.; Vogt, E.; Fromenteau, M.; Hortensteiner, S.; Matile, P.; Amrhein, N.; Martinoia, E. An ABC-transporter of Arabidopsis thaliana has both glutathione-conjugate and chlorophyll catabolite transport activity. Plant J. 1998, 13, 773-780.

65. Kispal, G.; Csere, P.; Prohl, C.; Lill, R. The mitochondrial proteins Atm1p and Nfs1p are essential for biogenesis of cytosolic Fe/S proteins. Embo. J. 1999, 18, 3981-3989.

66. Zolman, B.K; Silva, I.D.; Bartel, B. The Arabidopsis pxa1 mutant is defective in an ATP-binding cassette transporter-like protein required for peroxisomal fatty acid beta-oxidation. Plant Physiol. 2001, 127, 1266-1278.

67. Ruzicka, K.; Strader, L.C.; Bailly, A.; Yang, H.; Blakeslee, J.; Langowski, L.; Nejedla, E.; Fujita, H.; Itoh, H.; Syono, K.; Hejatko, J.; Gray, W.M.; Martinoia, E.; Geisler, M.; Bartel, B.; Murphy, A.S.; Friml, J. Arabidopsis PIS1 encodes the ABCG37 transporter of auxinic compounds including the auxin precursor indole-3-butyric acid. Proc. Natl. Acad. Sci. USA 2010, 107, 10749-10753. 
68. Geisler, M.; Blakeslee, J.J.; Bouchard, R.; Lee, O.R.; Vincenzetti, V.; Bandyopadhyay, A.; Titapiwatanakun, B.; Peer, W.A.; Bailly, A.; Richards, E.L.; Ejendal, K.F.; Smith, A.P.; Baroux, C.; Grossniklaus, U.; Muller, A.; Hrycyna, C.A.; Dudler, R.; Murphy, A.S.; Martinoia, E. Cellular efflux of auxin catalyzed by the Arabidopsis MDR/PGP transporter AtPGP1. Plant J. 2005, 44, 179-194.

69. Strader, L.C.; Bartel, B. The Arabidopsis PLEIOTROPIC DRUG RESISTANCE8/ABCG36 ATP binding cassette transporter modulates sensitivity to the auxin precursor indole-3-butyric acid. Plant Cell 2009, 21, 1992-2007.

70. Kuromori, T.; Miyaji, T.; Yabuuchi, H.; Shimizu, H.; Sugimoto, E.; Kamiya, A.; Moriyama, Y.; Shinozaki, K. ABC transporter AtABCG25 is involved in abscisic acid transport and responses. Proc. Natl. Acad. Sci. USA 2010, 107, 2361-2366.

71. Kang, J.; Hwang, J.U.; Lee, M.; Kim, Y.Y.; Assmann, S.M.; Martinoia, E.; Lee, Y. PDR-type ABC transporter mediates cellular uptake of the phytohormone abscisic acid. Proc. Natl. Acad. Sci. USA 2010, 107, 2355-2360.

72. Suh, S.J.; Wang, Y.F.; Frelet, A.; Leonhardt, N.; Klein, M.; Forestier, C.; Mueller-Roeber, B.; Cho, M.H.; Martinoia, E.; Schroeder, J.I. The ATP binding cassette transporter AtMRP5 modulates anion and calcium channel activities in Arabidopsis guard cells. J. Biol. Chem. 2007, 282, 1916-1924.

73. Lee, M.; Choi, Y.; Burla, B.; Kim, Y.Y.; Jeon, B.; Maeshima, M.; Yoo, J.Y.; Martinoia, E.; Lee, $\mathrm{Y}$. The $\mathrm{ABC}$ transporter AtABCB14 is a malate importer and modulates stomatal response to CO2. Nat. Cell Biol. 2008, 10, 1217-1223.

74. Pighin, J.A.; Zheng, H.; Balakshin, L.J.; Goodman, I.P.; Western, T.L.; Jetter, R.; Kunst, L.; Samuels, A.L. Plant cuticular lipid export requires an ABC transporter. Science 2004, 306, 702-704.

75. McFarlane, H.E.; Shin, J.J.; Bird, D.A.; Samuels, A.L. Arabidopsis ABCG transporters, which are required for export of diverse cuticular lipids, dimerize in different combinations. Plant Cell 2010, 22, 3066-3075.

76. Jasinski, M.; Stukkens, Y.; Degand, H.; Purnelle, B.; Marchand-Brynaert, J.; Boutry, M. A plant plasma membrane ATP binding cassette-type transporter is involved in antifungal terpenoid secretion. Plant Cell 2001, 13, 1095-1107.

77. Goossens, A.; Hakkinen, S.T.; Laksso, I.; Oksman-Caldentey, K.M.; Inze, D. Secretion of secondary metabolites by ATP-binding cassette transporters in plant cell suspension cultures. Plant Physiol. 2003, 131, 1161-1164.

78. Shitan, N.; Bazin, I.; Dan, K.; Obata, K.; Kigawa, K.; Ueda, K.; Sato, F.; Forestier, C.; Yazaki, K. Involvement of CjMDR1, a plant multidrugresistance-type ATP-binding cassette protein, in alkaloid transport in Coptis japonica. Proc. Natl. Acad. Sci. USA 2003, 100, 751-756.

79. Shitan, N.; Yazaki, K. Accumulation and membrane transport of plant alkaloids. Curr. Pharm. Biotechnol. 2007, 8, 244-252.

80. Baerson, S.R.; Sanchez-Moreiras, A.; Pedrol-Bonjoch, N.; Schulz, M.; Kagan, I.A.; Agarwal, A.K.; Reigosa, M.J.; Duke, S.O. Detoxification and transcriptome response in Arabidopsis seedlings exposed to the allelochemical benzoxazolin-2(3H)-one. J. Biol. Chem. 2005, 280, 21867-21881. 
81. Zhao, J.; Dixon, R.A. The 'ins' and 'outs' of flavonoid transport. Trends Plant Sci. 2010, 15, 72-80.

82. Klein, M.; Martinoia, E.; Hoffmann-Thoma, G.; Weissenbock, G. A membrane-potential dependent $\mathrm{ABC}$-like transporter mediates the vacuolar uptake of rye flavone glucuronides: regulation of glucuronide uptake by glutathione and its conjugates. Plant J. 2000, 21, 289-304.

83. Frangne, N.; Eggmann, T.; Koblischke, C.; Weissenbo“ck, G.; Martinoia, E.; Klein, M. Flavone glucoside uptake into barley mesophyll and Arabidopsis cell culture vacuoles. Energization occurs by $\mathrm{H}+$-antiport and ATP-binding cassettetype mechanisms. Plant Physiol. 2002, 128, 726-733.

84. Goodman, C.D.; Casati, P.; Walbot, V. A multidrug resistance-associated protein involved in anthocyanin transport in Zea mays. Plant Cell 2004, 16, 1812-1826.

85. Sugiyama, A.; Shitan, N.; Yazaki, K. Involvement of a soybean ATP-binding cassette-type transporter in the secretion of genistein, a signal flavonoid in legume-Rhizobium symbiosis. Plant Physiol. 2007, 144, 2000-2008.

86. Wink, M. Compartmentation of secondary metabolites and xenobiotics in plant vacuoles. In The Plant Vacuole; Leigh, R.A., Sanders, D., Eds.; Academic Press: San Diego, CA, USA, 1997; pp. 141-169.

87. Yazaki, K. Transporters of secondary metabolites. Curr. Opin. Plant Biol. 2005, 8, 301-307.

88. Diener, A.C.; Gaxiola, R.A.; Fink, G.R. Arabidopsis ALF5, a Multidrug Efflux Transporter Gene Family Member, Confers Resistance to Toxins. Plant Cell 2001, 13, 1625-1637.

89. Li, L.; He, Z.; Pandey, G.K.; Tsuchiya, T.; Luan, S. Functional Cloning and Characterization of a Plant Efflux Carrier for Multidrug and Heavy Metal Detoxification. J. Biol. Chem. 2002, 277, 5360-5368.

90. Debeaujon, I.; Peeters, A.J.M.; Leon-Kloosterziel, K.M.; Korneef, M. The TRANSPARENT TESTA 12 gene of Arabidopsis encodes a multidrug secondary transporter-like protein required for flavonoid sequestration in vacuoles of the seed coat endothelium. Plant Cell 2001, 13, 853-871.

91. Marinova, K.; Pourcel, L.; Weder, B.; Schwarz, M.; Barron, D.; Routaboul, J.M.; Debeaujon, I.; Klein, M. The Arabidopsis MATE transporter TT12 acts as a vacuolar flavonoid $/ \mathrm{H}^{+}$-antiporter active in proanthocyanidin-accumulating cells of the seed coat. Plant Cell 2007, 19, 2023-2038.

92. Zhao, J.; Dixon, R.A. MATE Transporters Facilitate Vacuolar Uptake of Epicatechin 3'-OGlucoside for Proanthocyanidin Biosynthesis in Medicago truncatula and Arabidopsis. Plant Cell 2009, 21, 2323-2340.

93. Gomez, C.; Terrier, N.; Torregrosa, L.; Vialet, S.; Fournier-Level, A.; Verries, C.; Souquet, J.M.; Mazauric, J.P.; Klein, M.; Cheynier, V.; Ageorges, A. Grapevine MATE-type proteins act as vacuolar $\mathrm{H}^{+}$-dependent acylated anthocyanin transporters. Plant Physiol. 2009, 150, 402-415.

94. Shoji, T.; Inai, K.; Yazaki, Y.; Sato, Y.; Takase, H.; Shitan, N.; Yazaki, K.; Goto, Y.; Toyooka, K.; Matsuoka, K.; Hashimoto, T. Multidrug and toxic compound extrusion-type transporters implicated in vacuolar sequestration of nicotine in tobacco roots. Plant Physiol. 2009, 149, 708-718.

95. Morita, M.; Shitan, N.; Sawada, K.; Van Montagu, M.C.; Inze, D.; Rischer, H.; Goossens, A.; Oksman-Caldentey, K.M.; Moriyama, Y.; Yazaki, K. Vacuolar transport of nicotine is mediated by a multidrug and toxic compound extrusion (MATE) transporter in Nicotiana tabacum. Proc. Natl. Acad. Sci. USA 2009, 106, 2447-2452. 
96. Yazaki, K. ABC transporters involved in the transport of plant secondary metabolites. FEBS Lett. 2006, 580, 1183-1191.

97. Klein, M.; Weissenbock, G.; Dufaud, A.; Gaillard, C.; Kreuz, K.; Martinoia, E. Different energization mechanisms drive the vacuolar uptake of a flavonoid glucoside and a herbicide glucoside. J. Biol. Chem. 1996, 271, 29666-29671.

98. Bartholomew, D.M.; Van Dyk, D.E.; Lau, S.C.; O’Keefe, D.P.; Rea, P.A.; Viitanen, P.V. Alternate energy-dependent pathways for the vacuolar uptake of glucose and glutathione conjugates. Plant Physiol. 2002, 130, 1562-1572.

99. Walczak, H.A.; Dean, J.V. Vacuolar transport of the glutathione conjugate of trans-cinnamic acid. Phytochem. 2000, 53, 441-446.

100. Rea, P.A.; Li, Z.S.; Lu, Y.P.; Drozdowicz, Y.M.; Martinoia, E. From vacuolar GS-X pumps to multispecific ABC transporters. Annu. Rev. Plant Physiol. Plant Mol. Biol. 1998, 49, 727-760.

101. Kolukisaoglu, H.U.; Bovet, L.; Klein, M.; Eggmann, T.; Geisler, M.; Wanke, D.; Martinoia, E.; Schulz, B. Family business: the multidrug resistance related protein (MRP) ABC transporter genes in Arabidopsis thaliana. Planta 2002, 216, 107-119.

102. Badri, D.V.; Loyola-Vargas, V.M.; Broeckling, C.D.; DelaPena, C.; Jasinski, M.; Santelia, D.; Martinoia, E.; Sumner, L.W.; Banta, L.M.; Stermitz, F.; Vivanco, J.M. Altered profile of secondary metabolites in the root exudates of Arabidopsis ATP-binding cassette transporter mutants. Plant Physiol. 2008, 146, 762-771.

103. Miao, Y.C.; Liu, C.J. ATP-binding cassette-like transporters are involved in the transport of lignin precursors across plasma and vacuolar membranes Proc. Natl. Acad. Sci. USA 2011, in press.

Sample Availability: Not available.

(C) 2011 by the authors; licensee MDPI, Basel, Switzerland. This article is an open access article distributed under the terms and conditions of the Creative Commons Attribution license (http://creativecommons.org/licenses/by/3.0/). 